\title{
Hierarchical Network Architectures of Carbon Fiber Paper Supported Cobalt Oxide Nanonet for High-Capacity Pseudocapacitors
}

\author{
Lei Yang, ${ }^{\dagger}$ Shuang Cheng, ${ }^{\dagger}$ Yong Ding, Xingbao Zhu, Zhong Lin Wang, and Meilin Liu* \\ School of Materials Science and Engineering, Georgia Institute of Technology, 771 Ferst Drive, Atlanta, Georgia 30332-0245, United \\ States
}

\author{
Supporting Information
}

\begin{abstract}
We present a high-capacity pseudocapacitor based on a hierarchical network architecture consisting of $\mathrm{Co}_{3} \mathrm{O}_{4}$ nanowire network (nanonet) coated on a carbon fiber paper. With this tailored architecture, the electrode shows ideal capacitive behavior (rectangular shape of cyclic voltammograms) and large specific capacitance $(1124 \mathrm{~F} / \mathrm{g})$ at high charge/discharge rate $(25.34 \mathrm{~A} / \mathrm{g})$, still retaining $\sim 94 \%$ of the capacitance at a much lower rate of $0.25 \mathrm{~A} / \mathrm{g}$. The muchimproved capacity, rate capability, and cycling stability may be attributed to the unique hierarchical network structures, which improves electron/ion transport, enhances the kinetics of redox reactions, and facilitates facile stress relaxation during cycling.
\end{abstract}

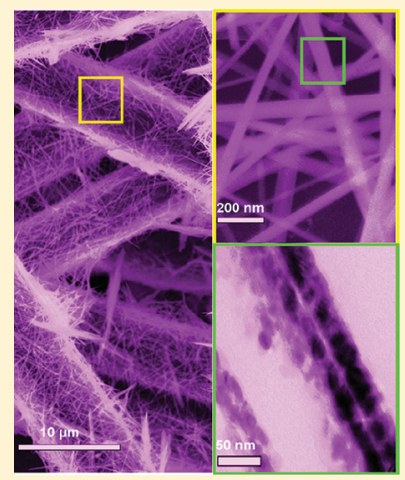

KEYWORDS: Pseudocapacitor, hierarchical network architecture, carbon fiber paper, rate capability

$\mathrm{E}$ lectrochemical capacitors are promising power sources for many portable systems and automotive applications due to their high power density, excellent pulse charge-discharge characteristics and long cycle life. ${ }^{1-4}$ Of all the metal oxides investigated as electrode materials for pseudocapacitor, hydrated ruthenium oxide is reported to be the most advantageous one, offering high specific capacitance with excellent cyclability. ${ }^{5}$ However, the high cost has impeded their usage in practical applications. A number of transition metal oxides have been studied as alternative electrodes such as $\mathrm{Co}_{3} \mathrm{O}_{4}, \mathrm{MnO}_{2}, \mathrm{~V}_{2} \mathrm{O}_{5}$, and $\mathrm{NiO}{ }^{6-9}$ To date, the pseudocapactiors with such electrodes invariably suffer from low capacitance, poor rate capability, and deterioration in performance associated with faradic redox reactions. ${ }^{10}$ Recently, oxide$\mathrm{Ag}$ (or $\mathrm{Au}$ ) hybrid electrodes were shown to improve specific capacitance and charge/discharge rate. ${ }^{11,12}$ For example, nanoporous $\mathrm{MnO}_{2}-\mathrm{Au}$ electrode ${ }^{11}$ demonstrated a specific capacitance of $\sim 170 \mathrm{~F} / \mathrm{g}$ at $20 \mathrm{~A} / \mathrm{g}$, which is $\sim 28.3 \%$ of the value at $0.3 \mathrm{~A} / \mathrm{g}$.

It has been well established that electrodes with proper nanostructures may enhance not only power density (or rate capability) but also cycling stability. ${ }^{1}$ While a wide variety of nanostructures have been created and tested, it still represents a grand challenge to identify the most promising structure or architecture that dramatically enhance the capacity while maintaining the excellent rate capability and charge-discharge cycling life. For example, cobalt oxides with a broad range of morphologies were successfully fabricated, including threedimensional (3D) oval-shaped microparticles, 2D nanosheets, and $1 \mathrm{D}$ needlelike nanorods. ${ }^{13}$ It was demonstrated that these porous $\mathrm{Co}_{3} \mathrm{O}_{4}$ structures exhibit enhanced initial specific capacity $(\sim 111 \mathrm{~F} / \mathrm{g})$, yet the rate capability and capacitance retention are still unsatisfactory. Nanonet, a random network of nanotubes, nanowires, or nanofibers, has been recently proposed as an advantageous architecture for transparent electrodes in optoelectronic devices due primarily to high transparency, better network conductance and fault tolerance. $^{14,15}$ Considering the similar requisite characteristics of electrodes in optoelectronic devices and in supercapactiors, we introduced oxide thin films with nanonet structures into supercapacitors in order to effectively enhance the specific capacitance and charge-discharge kinetics because the improved electron and ion percolation may enhance ionic and electronic transport through the electrode system.

Since many transition metal oxides exhibiting high pseudocapacitance have low electronic conductivity, these materials are often incorporated into highly conductive nanostructured carbons (e.g., carbon nanotube, graphene, or carbon aerogel) or directly deposited on metal foils. ${ }^{16-18}$ The use of carbon current collector and polymer binders (or additives) in the former approach adds extra cost, contact resistance, and weight. Furthermore, the large weight and limited electron path of metal foil supported electrodes may adversely affect the energy density. Low-cost carbon fiber paper (CFP), a network of microsized carbon fibers, has been extensively employed in proton exchange membrane fuel cells (PEMFC) as electrode substrate to support the catalyst,

Received: October 12, 2011

Revised: December 7, 2011 
conduct current, and allow gas transport. ${ }^{19}$ Because of the certain analogy in electrochemical reactions between supercapacitors and fuel cells, CFP may be a promising current collector and backbone for conformal coating of transition metal oxides for supercapactiors without using any insulating binders. Individual carbon fibers in CFP are well-connected and the conductive network and appropriate pore channels create an efficient electron percolation path as well as enable effective electrolyte access to the electrochemically active materials without limiting charge transport.

In this report, we present our findings on fabrication of a cobalt oxide nanonet thin film supported on a conductive carbon fiber network paper and demonstrate that such hierarchical nano/micro network architectures dramatically enhanced redox kinetics at high charge/discharge rates while maintaining electrochemical and structural stability.

Figure la shows some typical microstructures of the carbon fiber paper (from FuelCellStore) used in this study. The wellinterconnected carbon fibers with an average diameter of $\sim 5$

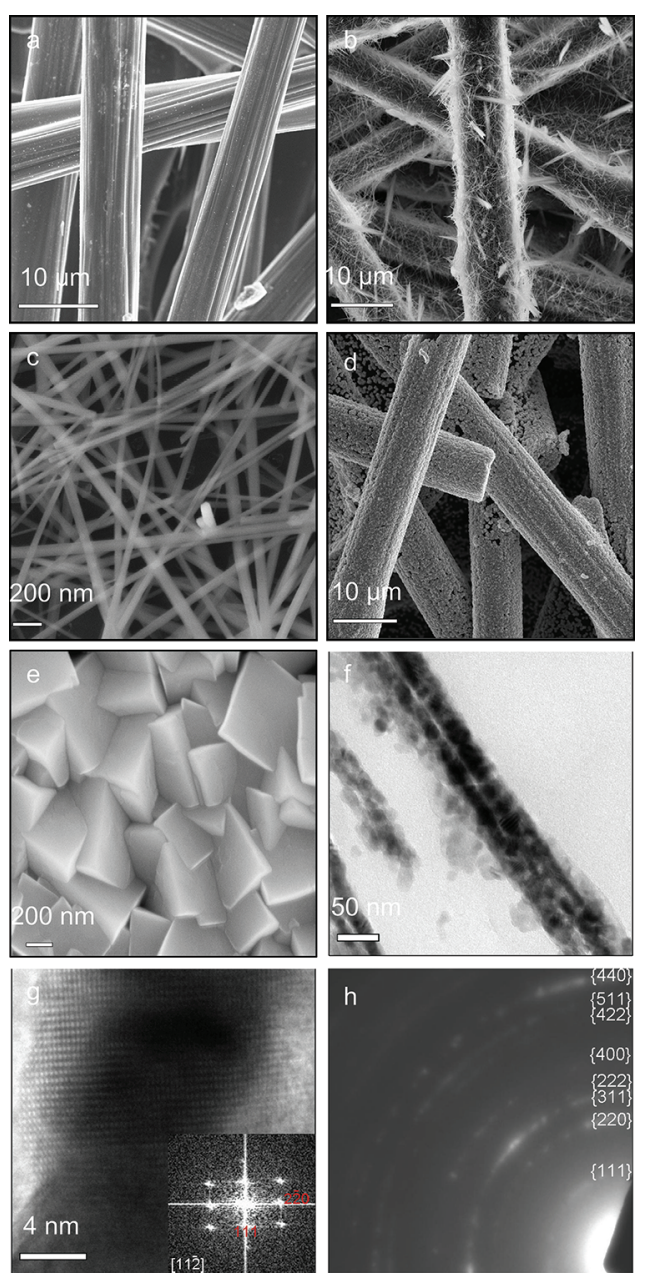

Figure 1. (a) SEM images of carbon fiber paper (CFP) before coating showing 3D network structures. (b) SEM image of carbon fiber paper after conformal coating of $\mathrm{Co}_{3} \mathrm{O}_{4}$ nanonet. (c) High-magnification SEM image of $\mathrm{Co}_{3} \mathrm{O}_{4}$ nanonet. (d) SEM image of carbon fiber paper after conformal coating of $\mathrm{Co}_{3} \mathrm{O}_{4}$ nanocube. (e) High-magnification SEM image of $\mathrm{Co}_{3} \mathrm{O}_{4}$ nanocube. (f) TEM image of $\mathrm{Co}_{3} \mathrm{O}_{4}$ nanowires. (g) HRTEM image of $\mathrm{Co}_{3} \mathrm{O}_{4}$ nanowires. Inset is corresponding fast Fourier transformation (FFT) pattern. (h) Diffraction pattern of $\mathrm{Co}_{3} \mathrm{O}_{4}$ nanowires. $\mu \mathrm{m}$ ensure sufficient mechanical strength and create an electrically conductive network. It is well established that the percolation geometry of a network is characterized by the ratio of stick areal density $D\left(\mathrm{~cm}^{-2}\right)$ to the percolation threshold density $D_{\text {perc }}$ described by eq $1^{20}$

$$
D_{\text {perc }}=\frac{4.236^{2}}{\pi L_{S}^{2}}
$$

where $L_{\mathrm{s}}$ is the length of the sticks and $D_{\text {perc }}$ is a critical number of sticks per unit area at which a stick network starts conducting globally; 4.236 is a numerical factor related to the "excluded volume" of the stick. The numerical factors for other geometrical shapes, for example, disks and squares, have values of 2.128 and 2.1, respectively. ${ }^{20}$ The discrepancy in percolation behaviors indicates that a stick network can use much fewer materials (or less weight) to maintain sufficient electron path. Therefore, the carbon fiber paper composed of numerous long carbon fibers is significantly advantageous for current collector and support of pseudocapacitive materials in lightweight supercapacitor toward portable and transport applications. Further, the pores formed between the microfibers may enable fast mass transport of electrolytes to all the surface of the electrochemically active materials, which is critical to high power density.

To identify which nanostructures are favorable for high-rate capacitive energy storage, we deposited $\mathrm{Co}_{3} \mathrm{O}_{4}$ with various morphologies directly onto carbon fiber papers using a facile hydrothermal synthesis route (see X-ray patterns in Supporting Information Figure S1). While the carbon fiber paper has a very complex porous structure, this in situ deposition technique readily produced thin, uniform coatings of $\mathrm{Co}_{3} \mathrm{O}_{4}$ on CFP, thereby retaining the network structure of the CFP. Also, the directly grown thin films can ensure good mechanical adhesion and electrical connection to the carbon fiber paper, avoiding the use of polymer binders and conducting additives (carbon or metal). Figure $1 \mathrm{~b}, \mathrm{c}$ shows the $\mathrm{Co}_{3} \mathrm{O}_{4}$ nanonet, similar to the carbon fiber network, creating an excellent electron and ion percolation path with high fault tolerance as well as numerous suitable pores for efficient ion access, which are more advantageous than the properties of $\mathrm{Co}_{3} \mathrm{O}_{4}$ nanocube coatings shown in Figure 1d,e. From the eq 1, the percolation threshold density $D_{\text {perc }}$ for nanonet and nanocube coatings are 0.0571 $\mathrm{Co}_{3} \mathrm{O}_{4}$ wires $/ \mu \mathrm{m}^{2}$ and $8.77 \mathrm{Co}_{3} \mathrm{O}_{4}$ cubes $/ \mu \mathrm{m}^{2}$, respectively, if we assume the average length of nanowires is $10 \mu \mathrm{m}$ and the width of square is $400 \mathrm{~nm}$. The large difference in the percolation threshold represents that under the same loading of active materials, the nanonet electrode possesses more electron/ion paths and faster transport of these charge carriers resulting from its high fault tolerance, possibly leading to higher mass specific capacitance and better rate capability. Therefore, $\mathrm{Co}_{3} \mathrm{O}_{4}$ nanonet supported on a carbon fiber paper with nano/ micro hierarchical network structures may be a promising binder-free electrode for supercapactiors. To investigate the detailed microstructure of $\mathrm{Co}_{3} \mathrm{O}_{4}$ nanonet, high-resolution TEM imaging and selected-area electron diffraction (SAED) analysis were employed. A typical TEM image of an individual $\mathrm{Co}_{3} \mathrm{O}_{4}$ nanowire is shown in Figure 1f. Consistent with the observation in SEM, the diameter of the $\mathrm{Co}_{3} \mathrm{O}_{4}$ nanowire is in the range of a few tens of nanometers. All the rings in the electron diffraction pattern in Figure $1 \mathrm{~h}$ recorded from the nanowires in Figure If can be uniquely indexed by the cubic 
structured $\mathrm{Co}_{3} \mathrm{O}_{4}$ (JCPDS No.00-042-1467, Fd3m, $a=\mathrm{b}=c=$ $0.808 \mathrm{~nm}$ ).

To evaluate the characteristic behavior of carbon fiber paper supported $\mathrm{Co}_{3} \mathrm{O}_{4}$ nanonet and nanocube electrodes for electrical energy storage, we performed cyclic voltammetry measurements at different potential scan rates (Figure $2 a-d$ ).
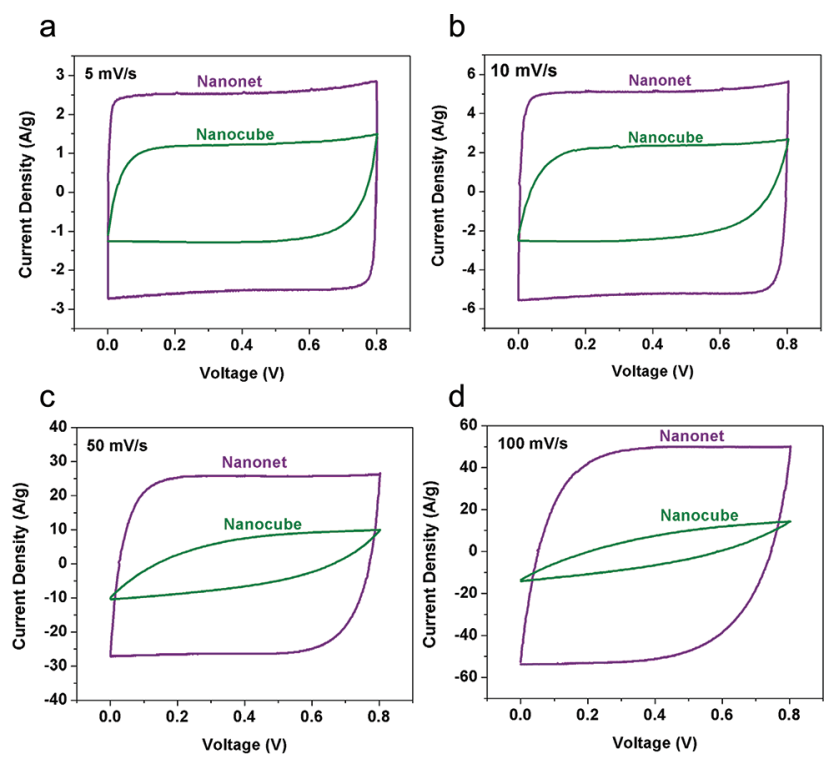

Figure 2. Cyclic voltammograms of CFP-supported $\mathrm{Co}_{3} \mathrm{O} 4$ nanonet and nanocube electrodes measured between $0-0.8 \mathrm{~V}$ at a scan rate of (a) 5, (b) 10, (c) 50, (d) $100 \mathrm{mV} / \mathrm{s}$. The CFP was shown to deliver negligible capacitance (see Supporting Information Figure S2).

Since the contribution of the carbon fiber paper to the capacitance is negligible (Supporting Information Figure S2), the total capacitance arises primarily from the redox pseudocapacitance of the loaded $\mathrm{Co}_{3} \mathrm{O}_{4}$. The absence of redox peaks in all the voltammograms indicates that the supercapacitors are charged and discharged at a pseudoconstant rate over the entire voltammetric cycles. ${ }^{11}$ At lower potential scan rates such as 5 and $10 \mathrm{mV} / \mathrm{s}$ (Figure 2a,b), the $\mathrm{Co}_{3} \mathrm{O}_{4}$ nanonet electrode showed perfectly symmetrical rectangular shapes with higher current density than nanocube electrode, representing an ideal capacitive behavior. Typically, the CV curves are significantly distorted with increasing potential scan rate, as evidenced by the nanocube electrode operated at 50 and $100 \mathrm{mV} / \mathrm{s}$ (Figure $2 \mathrm{c}, \mathrm{d}$ ). This is attributed mainly to the limited ion incorporation into the active electrode material; only the outer surface or subsurface is utilized for charge storage. ${ }^{21}$ In contrast, the nanonet electrode still retained excellent "rectangular-shape" cyclic voltammograms at 50 and $100 \mathrm{mV} / \mathrm{s}$, which appear even better than that for $\mathrm{Au}-\mathrm{MnO}_{2}$ electrode operated under similar conditions. ${ }^{11}$ To date, the most plausible elementary processes associated with the capacitive behavior of cobalt oxide can be described as follows ${ }^{22}$

$$
\begin{aligned}
& \mathrm{Co}_{3} \mathrm{O}_{4}+\mathrm{OH}^{-}+\mathrm{H}_{2} \mathrm{O} \Leftrightarrow 3 \mathrm{CoOOH}+e^{-} \\
& \mathrm{CoOOH}+\mathrm{OH}^{-} \Leftrightarrow \mathrm{CoO}_{2}+\mathrm{H}_{2} \mathrm{O}+e^{-}
\end{aligned}
$$

Therefore, rapid $\mathrm{OH}^{-}$intercalation/deintercalation and swift electron transfer are essential to achieve fast, reversible surface redox reactions. For the nanocube structure, its limited electron/ion path and lower fault tolerance at the same loading of active materials may limit the rate of the faradic reactions occurring on the interfaces. The nanonet structure could provide more electron/ion paths and faster transport of these charge carriers resulting from its high fault tolerance, thus leading to swift and reversible redox reactions as described by eqs 2 and 3 and hence to ideal rectangular CV curves (especially at low scan rates). In addition, the carbon fiber network serves as an efficient current collector. It is thus interesting to note that the nanocube electrode, despite the lower electrochemical performance than the nanonet electrode, yielded higher capacitance and better capacitive behavior than the previously reported $\mathrm{Co}_{3} \mathrm{O}_{4}$ electrodes. ${ }^{13,23,24}$ This implied that the embedded carbon fiber network may enhance not only the conductivity of the composite electrode but also the charge transfer rate of the $\mathrm{Co}_{3} \mathrm{O}_{4}$ electrodes due probably to improved interface between $\mathrm{Co}_{3} \mathrm{O}_{4}$ and carbon fiber during annealing. With such a hierarchical nano/micro network structures, the $\mathrm{Co}_{3} \mathrm{O}_{4}$ nanonet electrode supported on carbon fiber paper showed excellent capacitive behavior, while maintaining high capacitance at high cycling rates.

Further, we characterized the charge-discharge behaviors of the cobalt oxide nanonet and nanocube electrodes under galvanostatic conditions. As shown in Figure $3 a, b$, the
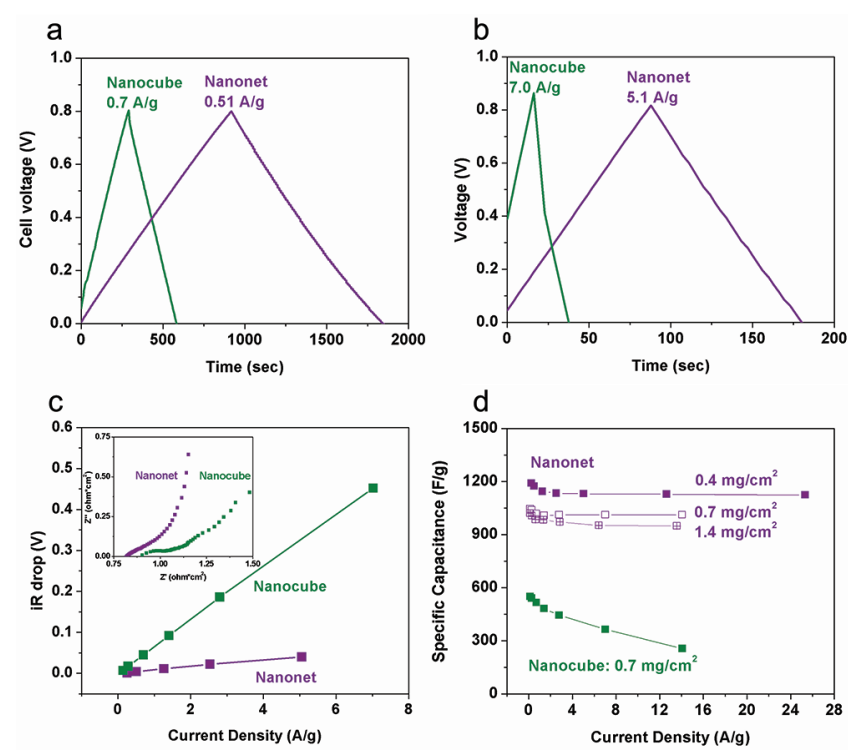

Figure 3. Typical charge-discharge curves of CFP-supported $\mathrm{Co}_{3} \mathrm{O}_{4}$ nanonet and nanocube electrodes at low (a) and high (b) constant currents. (c) $i R$ drop of the cells measured at different current densities. Inset shows electrochemical impedance spectra collected at open circuit voltage (OCV) under the influence of an ac voltage of 10 $\mathrm{mV}$. (d) Specific capacitances of CFP-supported $\mathrm{Co}_{3} \mathrm{O}_{4}$ nanonet (mass loading of $0.4,0.7$, and $1.4 \mathrm{mg} / \mathrm{cm}^{2}$ ) and nanocube electrodes (a mass loading of $0.7 \mathrm{mg} / \mathrm{cm}^{2}$ ) at different current densities. The mass loading of $1.4 \mathrm{mg} / \mathrm{cm}^{2}$ was obtained via laminating two CFP electrodes.

discharging time of the nanonet electrode was significantly increased in comparison with that of the nanocube electrode at both high and low current densities, suggesting that nanonet structure offered a much larger charge capacity, which was consistent with the CV curves. Moreover, the voltage-time profiles for the charging and discharging of the nanonet electrode were linear and symmetric, an indication of a high Coulombic efficiency and excellent reversibility. Conversely, the discharge profile of the nanocube electrode clearly consists of 
two parts: a resistive component arising from the sudden voltage drop representing the voltage change due to the equivalent series resistances (ESR) and a capacitive component related to the voltage change due to change in energy within the capacitor. Figure $3 c$ shows the $i R$ drops of the electrochemical capacitors as a function of applied current densities when the potentials were reversed (Supporting Information Figure S3). It is clearly seen that the nanonet electrode displayed much smaller ESR than the nanocube electrode at a given current density. This observation was further confirmed by the electrochemical impedance spectroscopy (EIS) measurements (insert of Figure 3c). It is noted that the ohmic
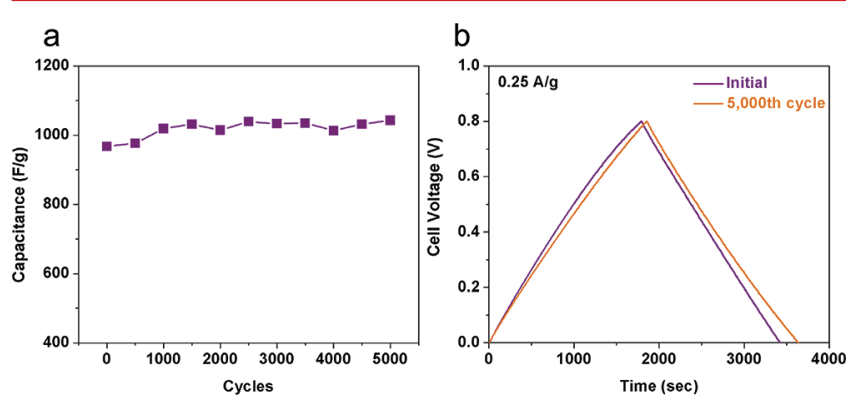

Figure 4. (a) Cycling performance of CFP-supported $\mathrm{Co}_{3} \mathrm{O}_{4}$ nanonet electrode $(5000 \mathrm{CV}$ cycles at a scan rate of $50 \mathrm{mV} / \mathrm{s})$, (b) chargedischarge curves before and after long-term cycling.

resistances of the two systems were similar, indicating that the interfacial contact between the cobalt oxide coatings and the carbon fibers were consistent. The smaller diameter of the semicircle in the impedance spectrum of the nanonet electrode was an indication of lower charge-transfer resistance. The internal resistance critically influences the power density or rate capability of electrochemical capacitors; low capacitance and deviation from ideal capacitor behavior of nanocube electrodes are primarily associated with large ESR. Comparing with nanocube structure, the nanonet enables an efficient percolation for both electrons and ions, leading to fast redox reactions and thus reduced internal resistance.

Figure $3 \mathrm{~d}$ compares the specific capacitances of nanonet and nanocube electrodes as a function of the applied current densities (calculated from the discharge curve). In the current density range investigated, the nanonet electrode yielded substantially higher specific capacitances than nanocube electrode. For example, at a mass loading of $0.4 \mathrm{mg} / \mathrm{cm}^{2}$, the specific capacitance for the nanonet electrode was $1190 \mathrm{~F} / \mathrm{g}$ at $0.25 \mathrm{~A} / \mathrm{g}$, which was more than twice that of the nanocube electrode $(540 \mathrm{~F} / \mathrm{g}$ at $0.28 \mathrm{~A} / \mathrm{g})$ and much higher than the values reported in the literatures. ${ }^{12,13,23,24}$ Also, the capacitance of the nanocube electrode dropped to $255 \mathrm{~F} / \mathrm{g}$ when the current density was increased to $14.07 \mathrm{~A} / \mathrm{g}$, as typically observed by others. ${ }^{11,12}$ This is attributed mainly to limited ion incorporation into the nanocube electrode material, resulting in a rapid increase in resistance to charge transport with increasing current density during cycling. However, the specific capacitance of the nanonet electrode was not kinetically limited and remained relatively constant at very high current densities, for example, reaching $1124 \mathrm{~F} / \mathrm{g}$ at $25.34 \mathrm{~A} / \mathrm{g}$, which was $\sim 94.4 \%$ of the value at $0.25 \mathrm{~A} / \mathrm{g}$. The excellent rate capability is superior to those of $\mathrm{Co}_{3} \mathrm{O}_{4}$ electrodes ever reported, even better than that of the $\mathrm{Co}_{3} \mathrm{O}_{4}-\mathrm{Ag}$ hybrid electrode. ${ }^{12}$ When the mass loading was increased to 0.7 and $1.4 \mathrm{mg} / \mathrm{cm}^{2}$, the specific capacitance of the nanonet electrode was reduced, respectively, to $\sim 1011$ and $\sim 948 \mathrm{~F} / \mathrm{g}$ at $14.06 \mathrm{~A} / \mathrm{g}$, still retaining relatively high specific capacitance at excellent rate capability. The results suggest that this hierarchical architecture is flexible in areal capacity and is ideally suited for fast and efficient energy storage. ${ }^{25}$ Obviously, the diffusion of the cations in the $\mathrm{Co}_{3} \mathrm{O}_{4}$ layer and the transport of electrons would affect the rate of the charge/discharge process. In our case, the thin nanonet layer with improved electron/ion percolation and a short diffusion path, together with the conductive carbon fiber network, can indeed provide an ideal pathway for ion and electron transport without kinetic limitations.

To evaluate the durability of the CFP-supported $\mathrm{Co}_{3} \mathrm{O}_{4}$ nanonet electrodes, we used cyclic voltammetry to characterize the long-term charge/discharge behavior at a potential scan rate of $50 \mathrm{mV} / \mathrm{s}$. Unlike most $\mathrm{Co}_{3} \mathrm{O}_{4}$ electrodes reported previously, ${ }^{12,26}$ the nanonet electrode displayed remarkable electrochemical stability up to 5000 cycles (Figure 4a). Similar to the $\mathrm{Cu}$ nanofiber network reported as a transparent electrode in optoelectronics, which could maintain their continuous network structures for conductance while releasing the strain built up during stretching, ${ }^{15}$ our hierarchical network structure appears able to tolerate the stress and/or volume change associated with reversible intercalation and/or adsorption of charge carrier. The facile stress relaxation of the tailored structure at the nanoscale effectively enhanced the electrochemical stability. Also, the specific capacitance experienced a slight increase after 5000 cycles. The improvement is further confirmed by the time-voltage profile for charging and discharging at a current density of $0.25 \mathrm{~A} / \mathrm{g}$ before and after the long-term cycling (Figure $4 \mathrm{~b}$ ). In particular, the chargedischarge curve became more linear and symmetric, due most likely to the improved charge transfer across the surface and interface.

In conclusion, we have created a hierarchical micro/nano network structure consisting of CFP coated with $\mathrm{Co}_{3} \mathrm{O}_{4}$ nanonet for supercapacitors. With this tailored architecture, the electrode shows high specific capacitance while maintaining an ideal capacitive behavior: high rate capability and excellent cycling stability, making it one of the best electrode systems for high-performance, lightweight supercapacitors. Further, the electrode design concept can be readily applied to other electrode materials (e.g., $\mathrm{MnO}_{2}$ and $\mathrm{V}_{2} \mathrm{O}_{5}$ ) for supercapacitor and battery applications.

\section{ASSOCIATED CONTENT}

\section{S Supporting Information}

Experimental details for the preparation and characterization of materials, components, and devices and additional supporting data. This material is available free of charge via the Internet at http://pubs.acs.org.

\section{AUTHOR INFORMATION}

\section{Corresponding Author}

*E-mail: meilin.liu@mse.gatech.edu

\section{Author Contributions}

${ }^{\dagger}$ These authors contributed equally.

\section{ACKNOWLEDGMENTS}

This material was based upon work supported as part of the HeteroFoaM Center, an Energy Frontier Research Center funded by the U.S. Department of Energy (DOE), Office of 
Science, Office of Basic Energy Sciences (BES) under Award Number DE-SC0001061.

\section{REFERENCES}

(1) Simon, P.; Gogotsi, Y. Nat. Mater. 2008, 7 (11), 845-854.

(2) Brezesinski, T.; Wang, J.; Tolbert, S. H.; Dunn, B. Nat. Mater. 2010, 9 (2), 146-151.

(3) Kim, J. H.; Zhu, K.; Yan, Y. F.; Perkins, C. L.; Frank, A. J. Nano Lett. 2010, 10 (10), 4099-4104.

(4) Hall, P. J.; Mirzaeian, M.; Fletcher, S. I.; Sillars, F. B.; Rennie, A. J. R.; Shitta-Bey, G. O.; Wilson, G.; Cruden, A.; Carter, R. Energy Environ. Sci. 2010, 3 (9), 1238-1251.

(5) Hu, C. C.; Chang, K. H.; Lin, M. C.; Wu, Y. T. Nano Lett. 2006, 6 (12), 2690-2695.

(6) Kim, H. K.; Seong, T. Y.; Lim, J. H.; Cho, W. I.; Yoon, Y. S. J. Power Sources 2001, 102 (1-2), 167-171.

(7) Kim, J. H.; Lee, K. H.; Overzet, L. J.; Lee, G. S. Nano Lett. 2011, 11 (7), 2611-2617.

(8) Chen, Z.; Qin, Y. C.; Weng, D.; Xiao, Q. F.; Peng, Y. T.; Wang, X. L.; Li, H. X.; Wei, F.; Lu, Y. F. Adv. Funct. Mater. 2009, 19 (21), $3420-3426$.

(9) Nam, K. W.; Yoon, W. S.; Kim, K. B. Electrochim. Acta 2002, 47 (19), 3201-3209.

(10) Jayalakshmi, M.; Balasubramanian, K. Int. J. Electrochem. Sci. 2008, 3 (11), 1196-1217.

(11) Lang, X. Y.; Hirata, A.; Fujita, T.; Chen, M. W. Nat. Nanotechnol. 2011, 6 (4), 232-236.

(12) Cheng, H.; Lu, Z. G.; Deng, J. Q.; Chung, C. Y.; Zhang, K. L.;

Li, Y. Y. Nano Res. 2010, 3 (12), 895-901.

(13) Zhu, T.; Chen, J. S.; Lou, X. W. J. Mater. Chem. 2010, 20 (33), 7015-7020.

(14) Hu, L. B.; Hecht, D. S.; Gruner, G. Chem. Rev. 2010, 110 (10), 5790-5844.

(15) Wu, H.; Hu, L. B.; Rowell, M. W.; Kong, D. S.; Cha, J. J.; McDonough, J. R.; Zhu, J.; Yang, Y. A.; McGehee, M. D.; Cui, Y. Nano Lett. 2010, 10 (10), 4242-4248.

(16) Zheng, L.; Xu, Y.; Jin, D.; Xie, Y. J. Mater. Chem. 2010, 20 (34), $7135-7143$.

(17) Yu, G. H.; Hu, L. B.; Vosgueritchian, M.; Wang, H. L.; Xie, X.; McDonough, J. R.; Cui, X.; Cui, Y.; Bao, Z. N. Nano Lett. 2011, 11 (7), 2905-2911.

(18) Hou, Y.; Cheng, Y. W.; Hobson, T.; Liu, J. Nano Lett. 2010, 10 (7), 2727-2733.

(19) Zhang, X. J.; Shen, Z. M. Fuel 2002, 81 (17), 2199-2201.

(20) Pike, G. E.; Seager, C. H. Phys. Rev. B 1974, 10 (4), 1421-1434.

(21) Bao, L. H.; Zang, J. F.; Li, X. D. Nano Lett. 2011, 11 (3), 12151220.

(22) Barbero, C.; Planes, G. A.; Miras, M. C. Electrochem. Commun. 2001, 3 (3), 113-116.

(23) Cao, L.; Lu, M.; Li, H. L. J. Electrochem. Soc. 2005, 152 (5), A871-A875.

(24) Wang, D. W.; Wang, Q. H.; Wang, T. M. Inorg. Chem. 2011, 50 (14), 6482-6492.

(25) Wei, T. Y.; Chen, C. H.; Chang, K. H.; Lu, S. Y.; Hu, C. C. Chem. Mater. 2009, 21 (14), 3228-3233.

(26) Gao, Y. Y.; Chen, S. L.; Cao, D. X.; Wang, G. L.; Yin, J. L. J. Power Sources 2010, 195 (6), 1757-1760. 